\title{
Understanding Design Empowerment through ICT-based Platforms in European Cities
}

\author{
Ahmet Gün ${ }^{1}$, Yüksel Demir ${ }^{2}$, Burak Pak ${ }^{3}$ \\ ${ }^{1,2}$ Istanbul Technical University Faculty of Architecture ${ }^{3} \mathrm{KU}$ Leuven Faculty of \\ Architecture \\ 1,2\{ahmetgun|demiry\}@itu.edu.tr ${ }^{3}$ burak.pak@kuleuven.be
}

\begin{abstract}
The use of ICT-based participation tools in urban design has gained importance in the last decade. In order to enable the citizens and other stakeholders to participate in city-making processes, a wide range of ICT-enabled participatory tools, techniques, and applications have been developed.. Many studies have reported that the use of these participatory platforms has led to positive outcomes but the platforms 'potentials and limits for facilitating different levels of design empowerment still remain unknown. In this context, this study aims to determine how these platforms empower citizens' engagement and identify the key factors that can facilitate better participation practices. This research analyses 25 ICT-based participation platforms by focusing on 4 key criteria:(1) their objectives, (2) the design action phases in which they are designated to be used, (3) their desired levels of design empowerment, and (4) offered functions. Our study reveals that more than three-fourth of analysed platforms still complemented by off-line participation activities. Empowering citizens to design independently is still a challenging task so just three platforms (12\%) allow users to create their own plans and visions. Finally, we identify several influence factors for better ICT-based participatory design practices.
\end{abstract}

Keywords: citizen empowerment, participatory design, ICT-based participation, urban design

\begin{abstract}
INTRODUCTION
Citizen participation in design has come into prominence because of the paradigm shift took place in the 1960s. There are lots of terms used related to citizen participation in design such as 'collaborative design', 'user involvement in design', 'citizen engagement in design', 'civic participation in design' . We used Participatory Design (PD) term in this study. PD is defined as a design practice which involves differ-
\end{abstract}

ent non-designer groups, who have different backgrounds, experiences, interests and roles, in various collaborative design activities throughout the design process (Sanders et al, 2010: 195). Thus, people who are outside of the profession have chance to participate in design process.

The use of ICT-based participation tools and techniques for urban related issues, especially for city-making, has come into prominence in the last 
decade. There are many technology enabled participatory tools, techniques, applications have been developed in order to provide participation atmosphere to stakeholders. We use 'ICT-based participation platforms' as an umbrella term in this study and this term is used to describe the 'forums created to source, analyze, visualize, and share information, expertise and solutions to advance social causes and/or solve social and policy problems' (Desouza, 2014:26). These platforms not only provide suitable communication channels to citizens but they also offer several innovations so that citizens can tackle challenges of urban areas and express their needs, desires and thoughts about urban-related issues.

It is important to know the potentials and limitations of these platforms in order to carry out successful city-making and management processes. There are several research have examined ICT-based platforms to reveal the empowerment goals (Pak and Verbeke, 2014a), to identify types of mobile apps for citizen participation (Ertiö, 2015), to demonstrate their potentials (Falco \& Kleinhans, 2018). Although these studies determines the potentials and limitations of these platforms to some extent, there is still a need for a comprehensive study. Thus, the aims of this study are to update the findings on these platforms by determining their affordances and identify the key factors that contribute to the success of ICT-based participatory practices.

With these aims in mind, this study addresses the following research questions:

- What are the affordances, potentials and limitations of ICT-based platforms for design empowerment?

- What are the key factors in facilitating and implementing better practices in the future using ICT-based civic participation platforms?

The next section start with a background review of ICT-based participation platforms in urban development. In section 3, we will describe our research methodology and the results of our analysis of selected platforms. In Section 4, we will evaluate the research findings and identify key success factors for better practices in the future. The literature review and analysis results of this study are based on our previous study that has already been published (Gün, Demir and Pak, 2019). Due to the word limitations, this paper presents a shortened version of literature review and analysis results.

\section{ICT-BASED PARTICIPATORY DESIGN}

Recent advances in information and communication technologies (ICT) have not only transformed the devices used by citizens but they also transformed the social life of humanity. Citizen participation in public life has gained importance in the digital era because ICT opens new communication channels between informal actors such as citizens and formal actors such as municipal officials and government agencies. These new forms of collaborative social interaction have led to important changes in societies and they will shape the patterns of our future social life (Höffken \& Streich, 2013).

ICT opens new possibilities not only for policymakers but also for citizens by empowering and fostering their self-organization so it changes the pattern of public participation (Kleinhans, Van Ham, \& Evans-Cowley, 2015). Especially social media and Web 2.0 applications provide a lot of services in order that people can get involved in activities and share their interests and concerns (Bannon \& Ehn, 2012).

This study explores the affordances of ICT-based platforms. To determine the affordances of platforms, we use the term, design empowerment, instead of participation. In literature, a plethora of participation spectrums or ladders (Arnstein, 1969; Connor, 1988; Rocha, 1997) have been developed to classify participation practices. However, these classifications heavily focus on the power relations between participants and a process and they do not suffice to explain the particular independent participatory activities carried out by citizens or organizations (Pak \& Verbeke, 2014b). Thus, we use the design empowerment framework constructed by Senbel and Church (2011) for identifying the affordances of platforms. 
We also use the five higher instances of this framework as a set of selection criteria. Senbel and Church proposed six design empowerment instances by applying questions to the visualization media as following (2011, p.426):

- Information (1): What information is presented by the medium? Is it diverse and is it sufficiently transparent to be understood by community residents?

- Inspiration (2): Does the medium trigger a response stimulating action toward a neighbourhood vision or design?

- Ideation (3): Does the medium provide a mechanism for community residents to express their ideas about their home neighbourhoods?

- Inclusion (4): Does the medium facilitate the inclusion of community residents' ideas into the planning and neighbourhood design process?

- Integration (5): Does the medium facilitate the integration of community residents in the neighbourhood planning and design process?

- Independence (6): Does the medium enable community residents to develop their own plans?

Social media networks, web-based participation platforms, mobile participation applications, and digital participation games have been extensively used by community in the digital era. Even though the affordances and potentials of these tools are different they have several common features such as the use of geolocation data, the logic of crowdsourcing and the use of internet. Our study analyses two types of ICTbased tools, web-based participation platforms and mobile participation applications.

Web-based participatory platforms have several capabilities such as 2D and 3D geovisualization capability and offer some tools, e.g. Web 2.0 collaboration tools and interactive sketch tools, in order that participants can involve in urban projects in collaborative environments (Poorazizi, Steiniger, \& Hunter, 2015). These platforms provide an interactive and communication oriented urban design process and transform urban design process into an iterative and agile work process because they enable to employ knowledge and experiences of non-professionals, test public attitude and acceptances in early project stages (Münster et al., 2017). Thus, these platforms can shape the future of urban design and planning towards a data-driven, networked and agile process.

Many web-based platforms have been developed to empower citizens' participation in urban design issues. BlockByBlock, Unlimited Cities, IdeeParis, Smarticipate, ZO!City are some of the web-based platforms that have already been used to involve non-professionals in design and planning process. Online games also offer participants promising environments and they can be used to support learning processes in new ways. NextCampus, Participatory Chinatown, and Second Life are online games that offer playful environments where citizens can deliberate over urban issues and submit design and planning proposals.

Mobile devices use various network interfaces such as WLAN, Bluetooth, and GPS-receivers, and they provide combined services using voice annotation, picture capturing and geo-referencing (de Reuver, Stein, \& Hampe, 2013). One of the most important features of mobile devices is its portability so it removes barriers of access by allowing users to be 'online whenever and wherever' and facilitates the participation practices (Ertiö, 2015). They also facilitate instant, location-based interactions between stakeholders (Schröder, 2015). Thus, many mobile participation applications have been used for data collection and make interaction with the public in urban development processes (Evans-Cowley \& Kubinski, 2015).

Mobile participation applications vary by the actions they are used for. While some applications such as SantanderAR just provides real-time information to users, some problem reporting apps such as Sag's Wien and FixMyStreet, are used to identify urban 
problems. Apps such as FlashPoll enable the public to involve in municipal decision-making processes through location-based instant polling and opiniongiving activities (Schröder, 2014).

\section{UNDERSTANDING DESIGN EMPOWER- MENT THROUGH ICT-BASED PLATFORMS}

This study focused on 4 key criteria while making the analysis,: (1) The Design Phases, (2) The Objectives Pursued by the Platforms, (3) The Functions Offered by the Platforms, (4) Intended Levels of Design Empowerment. We did a literature review to discover what is already studied, and synthesize the basic concepts and key insights about the relevant platforms. We firstly compiled a database of 106 relevant platforms. As the focus of this paper is design empowerment, we eliminated the platforms which just provide one-sided information exchange. We identified 56 suitable platforms, then we filtered out the platforms used outside of Europe. We finally selected 25 platforms for making systematic analysis (Table 1). For each platform, we did a content analysis by reviewing written reports and crosschecked them by analysing these platforms and by using when possible. As our content analysis was unable to elucidate some critical issues, we conducted questionnaires and interviews with 7 project developers.

\section{The Design Phases}

In literature, many researchers (Boyko, Cooper, \& Davey, 2005; Carmona, 2014) identify the design phases for urban design practices but their classifications do not suffice to identify the design phases of technology-driven participatory design practices. Thus, we are able to determine five basic steps for classifying the phases of ICT-based participatory practices: (1) collecting data about the current situation, (2) ideation, (3) conceptual design, (4) design and (5) public inquiry about design alternatives.

Out of all platforms analysed, FixMyStreet, Sag's Wien, ePart.it and Tirana Ime can just be used during data collection phase, whereas CityLab010, Collaborare A Bologna, Decide Madrid, Get the Mayor,
NextZuerich, StadtMacher and ZO!City allow participant to involve in process only during ideation phase. Betri Reykjavik and BlockByBlock allow participants to act during ideation, conceptual design, design and public inquiry about design alternatives phases. Maptionnaire is the only platform that can be used during all design phases. The design phase is important criteria for classifying the platforms but it is not sufficient enough to show the affordances and potentials of platforms. Thus, it is essential to consider the other three criteria while making the analysis.

\section{The Objectives Pursued by the Platforms}

To meet people's needs and desires, improve the quality of urban areas and solve urban-related problems, ICT-based platforms pursue a wide range of objectives. While some platforms pursue just one objective, others pursue more than one objectives. Of the platforms, $56 \%(n=14)$ are used for collecting new ideas to improve the quality of urban life anywhere in the city, 36\% (n=9) are used for problem identification, $36 \%(n=9)$ are used for collecting design proposals for specific urban areas. 4 platforms (16\%) are used for crowdfunding, and one platform, DecideMadrid, used for participatory budgeting.

While BetriReykjavik, Get the Mayor, IdeeParis, MinStad, NextZuerich are used just for collecting new ideas to improve the quality of urban life, Finding Places, Flashpoll, Opendoors, Qua-kit, and Smarticipate are used for collecting design proposals for specific urban areas. ePart.it, FixMyStreet, Sag's Wien, and Tirana Ime are used only for problem identification. Other platforms used for multiple purposes. For example, ZO!City is used for carrying out crowdfunding activities and collecting design proposals for specific urban areas, DecideMadrid is used for collecting new ideas to improve the quality of urban life and participatory budgeting.

\section{The Offered Functions}

ICT-based platforms offer a wide variety of functions to enable the participants can deliberate over project issues, connect other stakeholders, express their needs and desires concerning urban develop- 
Table 1

The platforms analyzed in this study

\begin{tabular}{|c|c|c|c|c|}
\hline \multicolumn{5}{|l|}{ Platforms } \\
\hline * Betri Reyjavik & * CommonPlace & * FlashPoll & $*$ MinStad & * Smarticipate \\
\hline * BlockByBlock & * DecideMadrid & * Get the Mayor & $*$ NextZurich & * Stadt Macher \\
\hline * Carticipe & & * Harava & * Opendoors & * Tirana Ime \\
\hline * CityLab010 & * Finding Places & * IdeeParis & * Qua-kit & * Unlimited Cities \\
\hline $\begin{array}{l}\text { * Collaborare A } \\
\text { Bologna }\end{array}$ & * FixMyStreet & * Maptionnaire & * Sag's Wien & * zo!City \\
\hline
\end{tabular}

ment project. After analysing the platforms, we were able to specify the functions offered by the platforms: adding placemarks and descriptive text, tagging content based on pre-defined categories, drawing polygons on maps, designing 3D objects on existing models, commenting on other users' content, adding geolocated photos, voting and ranking options, organizing polls, visualizing or ranking collected data, and follow-up mechanisms (Table 2).

One of the critical functions offered by of platforms is follow-up mechanism which enable participants to follow whether their ideas or notifications used in process or not. As most of the platforms aim to collect feedback and ideas of users, $92 \%$ of platforms enable participants to add placemarks or tag content in one dimension. On the other hand $28 \%$ $(n=7)$ of platforms allow participants to draw polygons on maps in two dimension, only $20 \%(n=5)$ of them enable to design 3D objects on digital models in three dimension. While reporting apps such as FixMyStreet and Tirana Ime just allow users to notify their problems by adding placemarks, Qua-kit, Smarticipate and MinStad are the most comprehensive platforms which enable multiple options to make notifications, send feedback and submit design proposals. Moreover, our analysis revealed that offline participation methods such as referendums, workshops, design meetings and face-to-face interviews still in use. $76 \%$ of platforms are complemented by at least one kind of these activities to carry out better practices.

\section{Maximum Level of Aimed Design Empower- ment}

As mentioned in the previous section, we use the five higher instances of design empowerment proposed by Senbel and Church (2011, p.426), which are Inspiration, Ideation, Inclusion, Integration, and Independence, to identify the affordance levels of analysed platforms because this framework addresses user media relations and applies questions to the capacity of visualization media. As some platforms afford participants more than one design empowerment level, we consider the maximum level of intended design empowerment to classify the platforms.

Of the platforms, only three platforms, MinStad, Maptionnaire, and Smarticipate, empower citizens at the highest level, Independence level, by enabling them to develop their own plans independently. While $24 \%$ of the platforms, e.g. BlockByBlock and Qua-kit, empower participants at Integration level by facilitating the integration of community residents in the design process, 32\% of platforms, such as CityLab010, Collaborare A Bologna, and FlashPoll empower citizens at the inclusion level by including ideas and thoughts of participants among other priorities in decision making process and $16 \%$ of them, NextZuerich, StadtMacher, Carticipe, and Commonplace, allow participants to express their ideas and thoughts about the future of their neighbourhoods. The rest of platforms (16\%) empower participants at the inspiration level, the lowest empowerment level, 


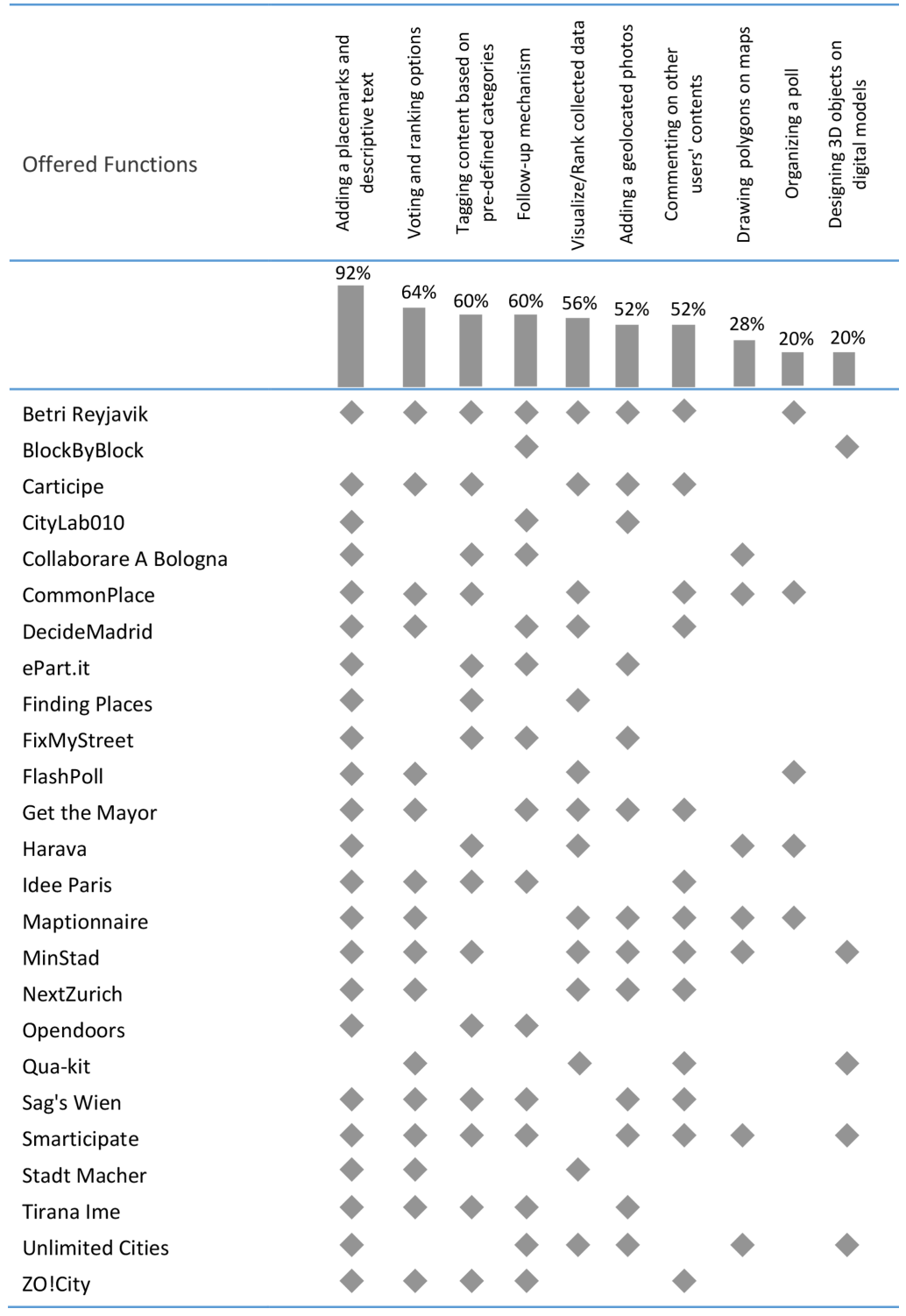

Table 2

The Offered

Functions 
by allowing them to act in response to the visualization material.

\section{DISCUSSION}

After analysing the platforms, we were able to make important findings concerning the affordances, potentials and limitations of the platforms. We revealed that there are a gap between the aimed level of design empowerment and actual level of design empowerment. $68 \%(n=17)$ of platforms are aimed to empower citizens at the middle and high design empoverment levels, inclusion, integration and independences, but $80 \%$ of them focus on doing basic functions such as feedback collection and geolocated annotations, that are correspond to the actions that can be done at the lowest levels of design empowerment, inspiration. The second important finding is related to the capabilities of users. While users can do basic actions such as tagging content, adding a placemark and descriptive text without in need of any help, there is a need to carry out extra activities when it comes to doing more complex actions such as designing objects on digital models. Thus, 76\% $(n=19)$ of platforms are complemented at least one traditional participation methods.

The third important finding concerns the relationship between follow-up mechanism and design empowerment level. As shown in the Table $2,60 \%$ of platforms enable users to follow whether their ideas and proposals are integrated into process or not. There is an inverse correlation between the level of design empowerment and the provision of follow-up mechanisms. While $100 \%$ of the platforms that empower citizens at the Inspiration level provide followup mechanism, only $33 \%$ of platforms that empower citizens at the independence level provide this function. Our study also revealed that majority of platforms still don't offer required design functions for users. $28 \%(n=7)$ of the platforms allow participants to draw polygons on maps and just $20 \%(n=5)$ of them enable participants to make collages or to design 3D objects on screen. These figures show us that although ICT-enabled participatory have progress in last years but there are still lacks and insufficiencies continue to exist related to providing suitable design environment.

\section{The Key Influence Factors for Better ICT- based Participatory Practices}

Equal representation between different interest group is one of the most essential issues in participatory design practices. Previous studies (Vicente \& Novo, 2014; Pak, Chua, \& Vande Moere, 2017) reported that as some interest groups are unable to access the internet to use these platforms, lack the capabilities to use them due to the social status, digital illiteracy, language barriers, ICT-based participatory practices can cause digital divides between different interest groups in community. Moreover, some community groups who already actively participate in society dominate the process and thus it cause exclusion problems (Bryson et al. 2013; Schröder, 2015). Unless disadvantages groups are eliminated to participate in process, the use of these platforms may enlarge the gap between the representation of different interest groups and thus cause new exclusion problems. Therefore, social, educational and technological background of community groups should be analysed before starting technology-driven participatory design processes. Then, participatory design activities, online or onsite, tools and additional training activities should be identified by considering the capabilities of all interest groups in community so that they can participate in process equally. Moreover, required outreach and promoting strategies should be determined in keeping with the attributes of different community groups to reach and inform them about process.

One of the most critical issues for implementing better participatory practices is providing suitable design environments for user experiences. Although $68 \%$ of the platforms are intended to empower citizens at the middle and high empowerment levels, the majority of platforms still focus on collecting citizens' ideas and notifications by allowing them to tag content based on pre-defined categories or 
add placemarks and descriptive text in one dimension. As explained above, only $20 \%$ of the platforms enable participants to submit 3D design proposals, and only $28 \%$ enable participants to draw polygons on maps. These figures show us that there are mismatches between the intended levels of design empowerment and the offered design environment for user experiences. Thus, suitable $2 \mathrm{D}$ and $3 \mathrm{D}$ design environments should be offered by platforms so that participants can develop and submit their design and planning ideas by varying criteria, e.g. public spaces, living, nature, and experience them in a virtual context. To do so, cutting edge technologies such as virtual reality and augmented reality should be used to provide high-level user experiences.

ICT-based participation platforms collect data from users and participants sometime submit proposals by using their private information. In some cases, such as MinStad, users can log on via their social media account. By considering the last discussions regarding data privacy issues, participants can feel that all of their private information and shared ideas are surveilled by the authorities or used by illintentioned groups so they may avoid from participating in process. Thus, all of the collected data should be protected carefully and users should be informed about how their shared and private information is used and by whom. Users' contributions should be anonymized except for carrying out collaborative group activities.

Providing follow-up mechanism is also one of the critical issues regarding trust. Our analysis revealed that there is an inverse correlation between the provision of follow-up mechanisms and the level of design empowerment. To provide trust and transparent process, participants should be informed about how their notifications and ideas integrated into design processes so all of platforms should provide followup mechanism. Visualizing and ranking collected data in an easy-to-understand format such as infographic and display it to users can remove the technical barriers so this action also contribute to increase the participants' engagement in design practices.

\section{CONCLUSION}

ICT-based platforms can change the future of urban development process towards data-driven, reflective and agile process. It is possible to involve large mass of people in urban design process, to use their knowledge and experiences and to test public reactions in early phases through these platforms (Münster et al., 2017). Moreover, these platforms provide more transparent communication channels so that formal actors such as government agencies and municipal officials and informal actors, such as citizens and neighbourhood associations can communicate and collaborate easily.

This study offered an overview of current ICTbased participation platforms and their actual use by stakeholders in urban development process by identifying the platforms' potentials and limitations for citizens' engagement. Moreover, we identify the key influence factors for better participatory practices. Although ICT-based participation platforms provide lots of opportunities for empowering citizens, they have yet to access their limits and do not suffice to carry out all participatory activities on their own due to the limitations explained in this study. There are just few platforms are able to provide suitable design environment for user experiences, and inform participants about what are the all activities in design processes and how users' shared information integrated into the design process. Therefore, these platforms should be considered as a complementary layer which increase the efficiency and effectiveness of other participation activities. Moreover, a lot of limitations continue to exist regarding trust, representation equality, and data privacy to afford citizens at the intended level of design empowerment in participatory design practices.

As the features and potentials of the platforms continue to progress, similar analysis study should be repeated. This study just addressed this issues based on declarative knowledge and no field observation was done. Thus, there is need to make indepth field study that analyze the reaction of users and other stakeholders to figure out the real impact 
on these platforms from the perspective of multiple stakeholders.

\section{REFERENCES}

Boyko, C. T., Cooper, R. and Davey, C. 2005 'Sustainability and the urban design process.', Proceedings of the Institution of Civil Engineers - Engineering Sustainability, p. $119-125$

Bryson, J. M., Quick, K. S., Slotterback, C. S. and Crosby, B. C. 2013, 'Designing public participation processes', Public Administration Review, 73(1), p. 23-34

Carmona, M. 2014, 'The place-shaping continuum: A theory of urban design process', Journal of Urban Design, 19(1), p. 2-36

Connor, D. M. 1988, 'A new ladder of citizen participation', National Civic Review, 77(3), p. 249-257

Desouza, K. C. and Bhagwatwar, A. 2014, 'Technologyenabled participatory platforms for civic engagement: The case of US cities', Journal of Urban Tech nology, 21(4), p. 25-50

Ertiö, T. P. 2015, 'Participatory apps for urban planning-space for improvement', Planning Practice \& Research, 30(3), p. 303-321

Evans-Cowley, J. S. and Kubinski, B. 2015, 'There's an App for that: Mobile applications that advance urban planning', in Silva, C. N. (eds) 2015, Emerging issues, challenges, and opportunities in urban E-planning, IGI Global, p. 33-45

Falco, E. and Kleinhans, R. 2018, 'Digital participatory platforms for co-production in urban development: A systematic review.', International Journal of E-Planning Research, 7(3), p. 1-21

Gün, A., Demir, Y. and Pak, B. 2019, 'Urban design empowerment through ICT-based platforms in Europe.', International Journal of Urban Sciences, 29, pp. 1-27

Höffken, S. and Streich, B. 2013, 'Mobile participation: Citizen engagement in urban planning via smartphones', in Silva, C. N. (eds) 2013, Citizen eparticipation in urban governance: Crowdsourcing and collaborative creativity, IGI Global., p. 199-225

Kleinhans, R., Van Ham, M. and Evans-Cowley, J. 2015, 'Using social media and mobile Technologies to foster engagement and self-organisation in participatory urban planning and neighbourhood governance', Planning Practice \& Research, 30(3), p 237-247

Münster, S., Georgi, C., Heijne, K., Klamert, K., Noennig, J. R., Pump, M. and van der Meer, H. 2017, 'How to involve inhabitants in urban design planning by using digital tools? An overview on a state of the art key challenges and promising approaches', Procedia Computer Science, 112, p. 2391-2405

Pak, B., Chua, A. and Vande Moere, A. 2017, 'Fixmystreet Brussels: Socio-demographic inequality in crowdsourced civic participation', Journal of Urban Technology, 24(2), p. 65-87

Pak, B. and Verbeke, J. 2014a 'Geoweb 2.0 and design empowerment: A critical evaluation of eleven cases', Digital Humanities 2014 Conference Proceedings

Pak, B. and Verbeke, J. 2014b, 'Geoweb 2.0 for participatory urban design: Affordances and critical success factors', International Journal of Architectural Computing, 12(3), p. 283-305

Poorazizi, M. E., Steiniger, S. and Hunter, A. J. 2015, 'A service-oriented architecture to enable participatory planning: An e-planning platform', International Journal of Geographical Information Science, 29(7), p. $1081-1110$

de Reuver, M., Stein, S. and Hampe, J. F. 2013, 'From eParticipation to mobile participation: Designing a service platform and business model for mobile participation', Information Polity, 18(1), p. 57-73

Rocha, E. M. 1997, 'A ladder of empowerment', Journal of Planning Education and Research, 17(1), p. 31-44

Sanders, E. B.-N., Brandt, E. and Binder, T. 2010 'A framework for organizing the tools and techniques of participatory design., Proceedings of the 11th biennial participatory design conference., pp. 195-198.

Schröder, C. 2014 'Mobile participation in urban development. International Reports on Socio-Informatics (IRSI)', Proceedings of the COOP 2014 Workshop on Collaborative Technologies in Democratic Processes, p. 37-44.

Schröder, C. 2015 'Through space and time: Using mobile apps for urban participation', Conference for edemocracy and open government, p. 133-142

Vicente, M. R. and Novo, A. 2014, 'An empirical analysis of e-participation. The role of social networks and egovernment over citizens' online engagement.', Government Information Quarterly, 31(3), p. 379-387 